\title{
A W-Band Waveguide Band-Stop Filter Using Electromagnetic Crystal (EMXT) Surface
}

\author{
Te-Chuan Chen, Hualiang Zhang, Hao Xin \\ Department of Electrical and Computer Engineering, University of Arizona, Tucson, AZ, USA \\ Email: hxin@ece.arizona.edu
}

How to cite this paper: Chen, T.-C., Zhang, H.L. and Xin, H. (2016) A W-Band Waveguide Band-Stop Filter Using Electromagnetic Crystal (EMXT) Surface. Circuits and Systems, 7, 4001-4008. http://dx.doi.org/10.4236/cs.2016.712331

Received: March 22, 2016

Accepted: April 20, 2016

Published: October 28, 2016

Copyright $\odot 2016$ by authors and Scientific Research Publishing Inc. This work is licensed under the Creative Commons Attribution International License (CC BY 4.0).

http://creativecommons.org/licenses/by/4.0/

\begin{abstract}
A rectangular waveguide band-stop filter using electromagnetic crystals (EMXT) surface as the top and bottom walls is presented in this letter. The EMXT surface has a band gap that can prevent electromagnetic wave from propagating along the waveguide. Dispersion properties of the waveguide filter are analyzed using an approximated lumped-element circuit model. A W-band prototype based on the proposed waveguide filter is designed and characterized. The experimental results show a rejection band centered at $97 \mathrm{GHz}$ and a rejection level of $16 \mathrm{~dB}$.
\end{abstract}

\section{Keywords}

W-Band, Band-Stop Filter, Electromagnetic Crystal, Band-Gap, Waveguide

\section{Introduction}

Electromagnetic crystals (EMXT), also known as electromagnetic band-gap (EBG) structures, are periodic structure that exhibits band-gap characteristics. The EMXT surface discussed here is a $2 \mathrm{D}$ artificial impedance surface that provides high impedance at its resonance. In general, the artificial impedance surface is consisted of periodic metal patches/strips on top of a thin substrate with complete metallization on the back. The substrate may include vias that connect the top metal patches/strips to the back ground plane. The grounded substrate provides inductive response that in conjunction with the capacitive patches/strips forms a resonant structure. Due to their unique properties, the high-impedance surfaces have been used in many applications such as antenna ground planes [1] [2], quasi-TEM waveguides [3] [4], band-pass filters [5] [6], and band-stop filters [7].

In this letter, a W-band band-stop filter using EMXT surfaces is presented. The filter is a rectangular waveguide with EMXT surface loaded on the top and/or bottom walls, 
which prevents wave propagation inside the waveguide due to its band-gap properties. Analysis of the unit cell response of the EMXT surface to obtain the band-gap information is commonly practiced. However, the band gap of the filter does not exactly equal to the band gap of the EMXT surface. The width and height of the waveguide will also influence the filter band gap. To further understand the overall response, a lumpedelement circuit model is introduced for the analysis of this waveguide band-stop filter. Eigen-mode and full-waveguide models using ANSYS HFSS are used to validate the equivalent circuit model. A prototype $\mathrm{W}$-band filter is designed and characterized with a stop band centered at $97 \mathrm{GHz}$ and a rejection level of $16 \mathrm{~dB}$.

\section{Filter Structure and Lumped-Element Circuit Model}

The design procedure of the EMXT surface general uses the unit cell method [3]. The unit cell method obtain the reflection properties of the surface as an infinite array for a normally incident wave. The reflection properties indicate a band gap in the surface when the reflection phase is near zero. Base on this band gap information, the designed EMXT surface implemented in the waveguide will yield a band-stop filter for this work. This information is sufficient; however, a more thorough analysis is needed to have a better understanding of the filter design. The objective here is to derive a design procedure from an approximated lumped element model based on the filter structure. The configuration of the waveguide band-stop filter is shown in Figure 1. Assuming wave propagation in the $\mathrm{z}$ direction, the air-filled rectangular waveguide has a waveguide width of $a$ in the $\mathrm{x}$ direction, and a waveguide height of $b$ in the y direction. The bottom wall of the waveguide is loaded with an EMXT surface. Figure 2 is the top view of the EMXT surface used. It is a thin dielectric substrate with the front side made of periodic metal strips and the backside completely metalized. The substrate is populated with vias that connect the metal strip to the backside ground plane to prevent undesired substrate waves. At the unit cell level, the capacitive coupling between the adjacent metal strips and the inductive response associated with the grounded substrate form a resonant structure. Due to the resonant nature of the EMXT surface, high surface impedance is observed at the resonant frequency.

To better understand the band-stop waveguide working principle, an equivalent circuit model is proposed as shown in Figure 3. The model is a cascade of the responses

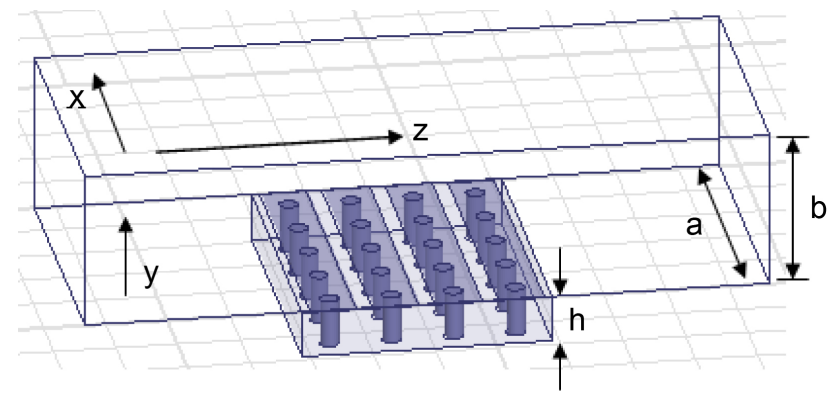

Figure 1. A waveguide band-stop filter with bottom wall loaded with EMXT surface. 


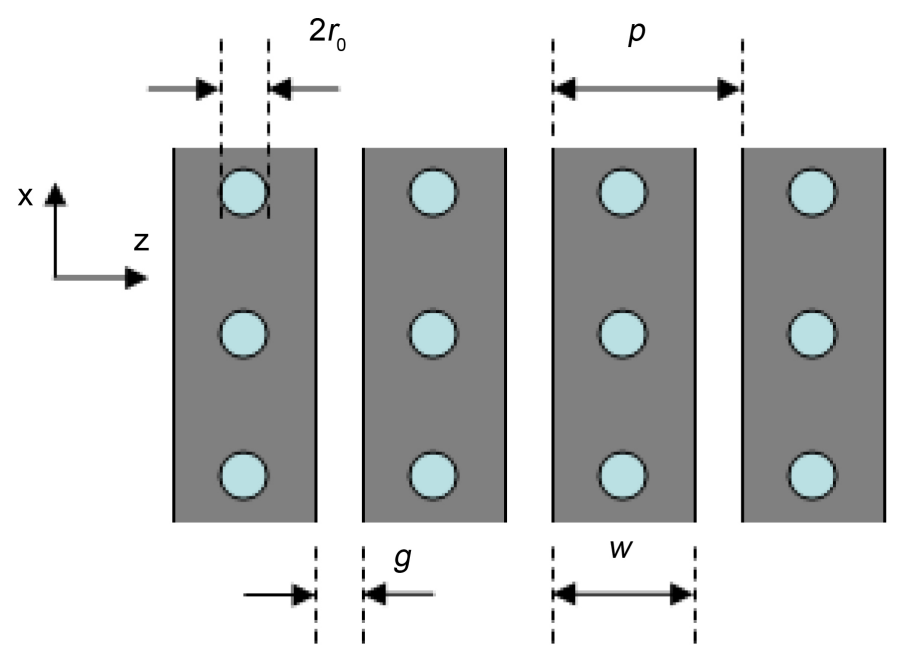

Figure 2. Top view of the EMXT surface.

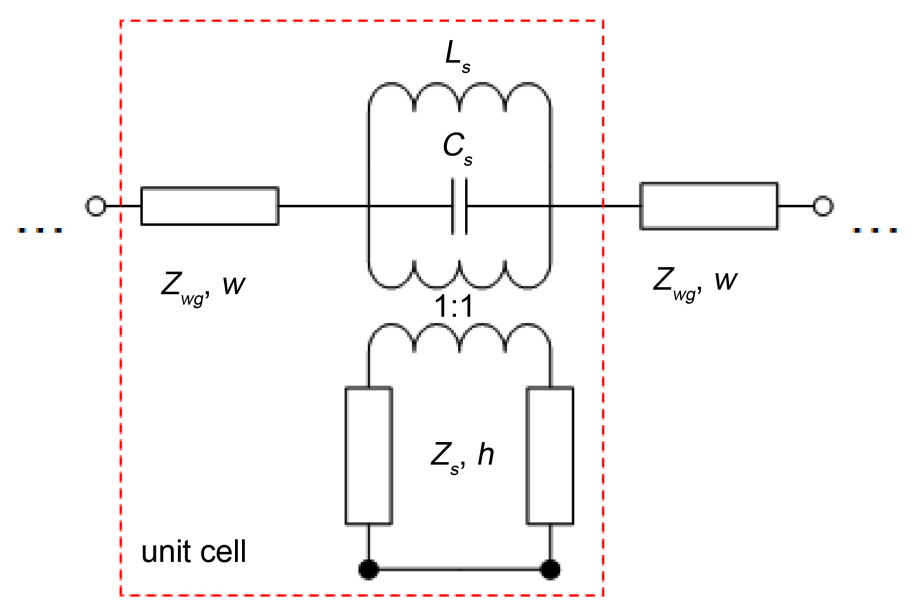

Figure 3. Lumped-element circuit model of the waveguide band-stop filter.

from the section of conventional rectangular waveguide and from the section loaded with EMXT surface. Inside the waveguide, the bottom wall alternates periodically between metal and non-metal surfaces. The metallic section behaves as a conventional rectangular waveguide. Using the transmission line equation, the dominant TE10 mode of a rectangular waveguide is expressed by the modal voltage and current.

$$
\frac{\mathrm{d} V}{\mathrm{~d} z}=-j \omega L I, \frac{\mathrm{d} I}{\mathrm{~d} z}=-j \omega C V
$$

where $L$ and $C$ are the per-unit-length series inductance and shunt capacitance of the transmission line model of the rectangular waveguide, respectively $\left(Z_{w g}=j \omega L+(j \omega C)^{-1}\right)$. They can be expressed as

$$
L=\mu \frac{Z_{0}}{\eta}, C=\varepsilon \frac{\eta}{Z_{0}}\left[1-\left(\frac{f_{c}}{f}\right)^{2}\right]
$$




$$
Z_{0}=\eta \frac{2 b}{a}
$$

where $f$ is the operating frequency, $f_{c}$ is the cutoff frequency of the $\mathrm{TE}_{10}$ mode, and $Z_{0}$ is the power-voltage definition of the characteristic impedance of a rectangular waveguide, as well as $\mu=\mu_{0}, \mathcal{E}=\varepsilon_{0}$, and $\eta=\eta_{0}$ assuming an air-filled waveguide. The non-metallic gap section is modeled as a parallel LC circuit, illustrated as $L_{s}$ and $C_{s}$ [8] in Figure 3, coupled to a grounded substrate by a transformer of 1:1 ratio. The model of the grounded substrate uses similar concept as a corrugated surface, where each gap section of the filter is approximated as a dielectric-filled groove [9]. Following this approximation, each gap section is modeled by treating the space between two rows of vias as a small waveguide with short termination. For this small waveguide, the dimensions $a, p$, and $h$ are its width, height and length, respectively, as illustrated in Figure 1 and Figure 2. The modeled $L_{s}$ and $C_{s}$ together with the small waveguide in the EMXT substrate forms admittance when they are placed in parallel. The admittance $Y_{g a p}$ of the non-metallic gap section is

$$
\begin{gathered}
Y_{\text {gap }}=j \omega C_{s}+\frac{1}{j \omega L_{s}}+\frac{1}{j Z_{s} \tan \beta_{s} h} \\
\beta_{s}=\sqrt{k_{s}^{2}-\left(\frac{\pi}{a}\right)^{2}}, k_{s}=\omega \sqrt{\varepsilon_{r} \varepsilon_{0} \mu_{0}}, Z_{s}=\frac{2 p}{a} \sqrt{\frac{\mu_{0}}{\varepsilon_{r} \varepsilon_{0}}} \frac{k_{s}}{\beta_{s}} .
\end{gathered}
$$

where $Z_{s}$ and $\beta_{s}$ are the characteristic impedance and propagation constant of the wave in the substrate of the gap section, respectively. Meanwhile, $h$ is the substrate thickness, $\mathcal{E}_{r}$ is the substrate dielectric constant, and $p$ is the unit cell periodicity. By applying the Bloch-Floquet theorem [10] to the unit cell, the dispersion relation of the waveguide filter is given by

$$
\begin{aligned}
& \beta(\omega)=\frac{1}{p} \cos ^{-1}\left(1+\frac{Z Y}{2}\right) \\
& Z=j \omega L+Y_{\text {gap }}^{-1}, Y=j \omega C
\end{aligned}
$$

where $Z$ is the series impedance and $Y$ is the shunt admittance of the unit cell. Additionally, using image symmetry, a single-walled EMXT filter can be used to model a double-walled EMXT filter of twice the waveguide height. This can reduce simulation size by a half while producing the same simulation results. As illustrated in Figure 4, $S$-parameters are compared between a single-walled filter at height $b$ and a doublewalled filter at height $2 b$ using EMXT surfaces with a length of eight unit cells $(8 p)$ instead of infinite length. The results have good agreement.

\section{Filter Design and Characterization}

The design parameters of an EMXT surface are substrate dielectric constant, substrate thickness, strip width and gap, substrate via radius and periodicity. Here the designed EMXT surface has the following parameters: $\mathcal{E}_{r}=2.2, h=0.381 \mathrm{~mm}, w=0.381 \mathrm{~mm}, g=$ $0.127 \mathrm{~mm}, r_{0}=0.076 \mathrm{~mm}$ and $p=0.508 \mathrm{~mm}$. For a WR-10 waveguide $(a=2.54 \mathrm{~mm}$ 


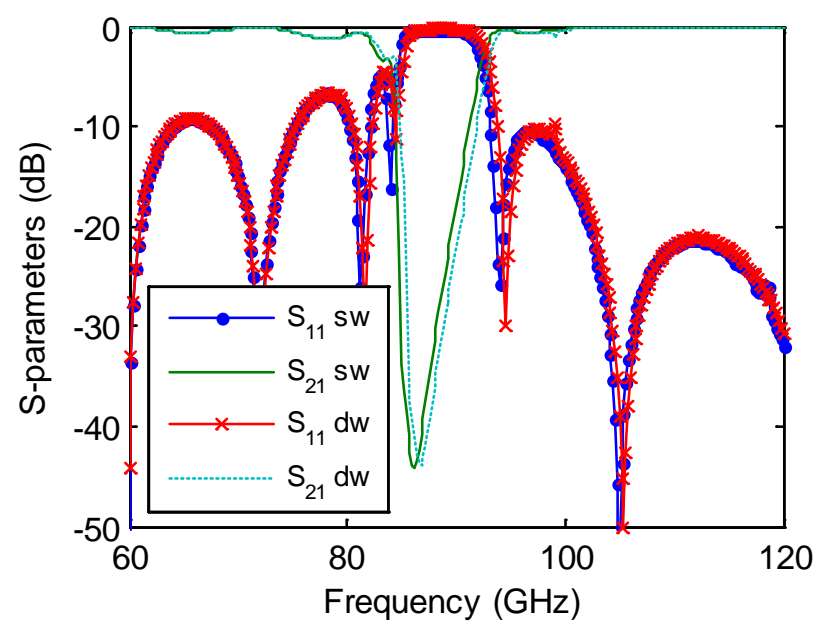

Figure 4. HFSS simulated $S$-parameters of a single-walled (sw) and a double-walled ( $\mathrm{dw}$ ) filter with waveguide height $b$ and $2 b$, respectively.

and $b=1.27 \mathrm{~mm}$ ) with the bottom wall loaded with an EMXT surface, the dispersion diagram of the filter is plotted in Figure 5. The comparable results of the circuit model and of the HFSS eigen-mode simulation both predict a band gap at $83-90 \mathrm{GHz}$. The light line (free-space propagation) is also plotted as a reference to the filter dispersion curves. As frequency increases beyond the plotted range, the dispersion curves should converge to the light line. In addition, the design equations of the circuit model can be used early in the design procedure to quickly calculate the filter response. Subsequently, the results from the circuit model calculation are later verified by the full-wave simulation model, which will likely to take longer time to complete.

To verify the design concept, a prototype waveguide filter is designed with a rejection band centered at $87 \mathrm{GHz}$. The filter is configured with both the top and the bottom walls loaded with EMXT surfaces, which is fabricated using Duroid 5880 substrate with 0.25 -ounce copper cladding. Due to the small dimension of the $\mathrm{W}$-band waveguide, a test fixture with a housing for the EMXT surface is fabricated, as shown in Figure 6. The internal dimension of the test fixture is shown in Figure 7. The test fixture is connected to WR-10 feeds at both input and output. A quarter-wave transformer section is used to match the WR-10 section to the EMXT filter section. The EMXT filter section holds EMXT surfaces with a length of four unit cells. $S$-parameter measurements are done using an Agilent E8361A Vector Network Analyzer with Agilent N5260A millimeter-wave controller after a full two-port WR-10 waveguide calibration at W-band (75 - $110 \mathrm{GHz}$ ). Figure 8 shows the test setup including the test fixture, VNA, and the W-band waveguides.

As shown in Figure 9 and Figure 10, the EMXT filter has a measured stopband centered at $97 \mathrm{GHz}$ spanning $94.7-99.4 \mathrm{GHz}$ (for $\left|S_{21}\right|<-10 \mathrm{~dB}$ ). Compared to the measured result, the HFSS simulated stopband is centered at $87 \mathrm{GHz}$, which is a $9.3 \%$ discrepancy in frequency. The measured rejection level is $16 \mathrm{~dB}$ at $97 \mathrm{GHz}$. Outside the 


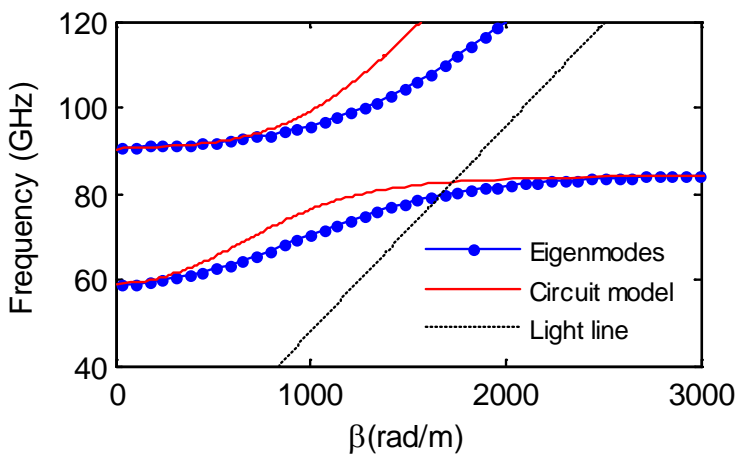

Figure 5. Dispersion diagram of the designed waveguide band-stop filter.

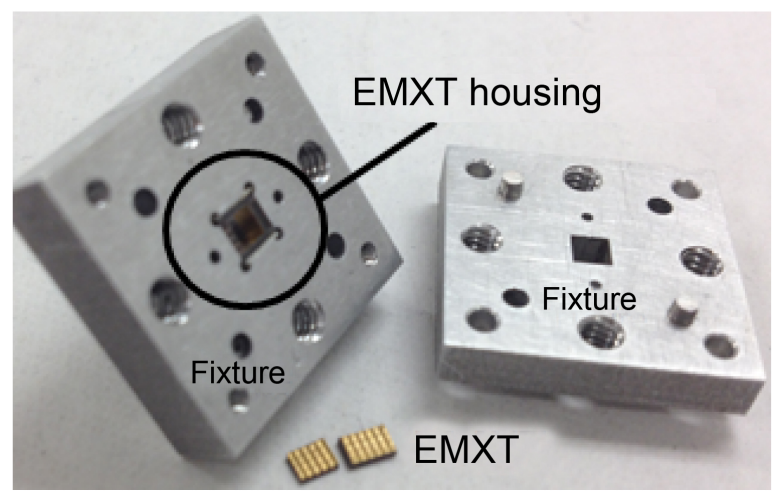

Figure 6. Test fixture and the EMXTs of the filter.

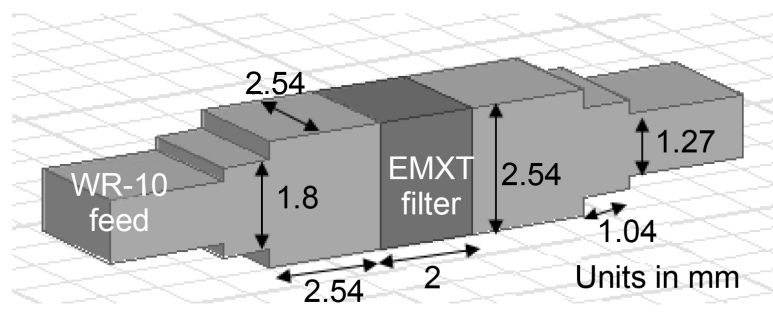

Figure 7. The internal housing of the test fixture.

stopband, the transmission loss is roughly 3 to $7 \mathrm{~dB}$. Large return loss up to $2.6 \mathrm{~dB}$ is measured in the stopband, as shown in Figure 8. Also, measured and simulated return loss has a similar frequency discrepancy. The deviation between the measurement and the simulation is most likely caused by assembly misalignment and manufacturing imperfection, especially in W-band which any small shift in the alignment or fabrication can result in a very significant shift in the frequency response.

\section{Conclusion}

A waveguide band-stop filter using EMXT surfaces has been investigated. The analysis by equivalent lumped-element circuit model shows good agreement with the full-wave simulation in predicting the filter stopband. A prototype EMXT filter has been success- 


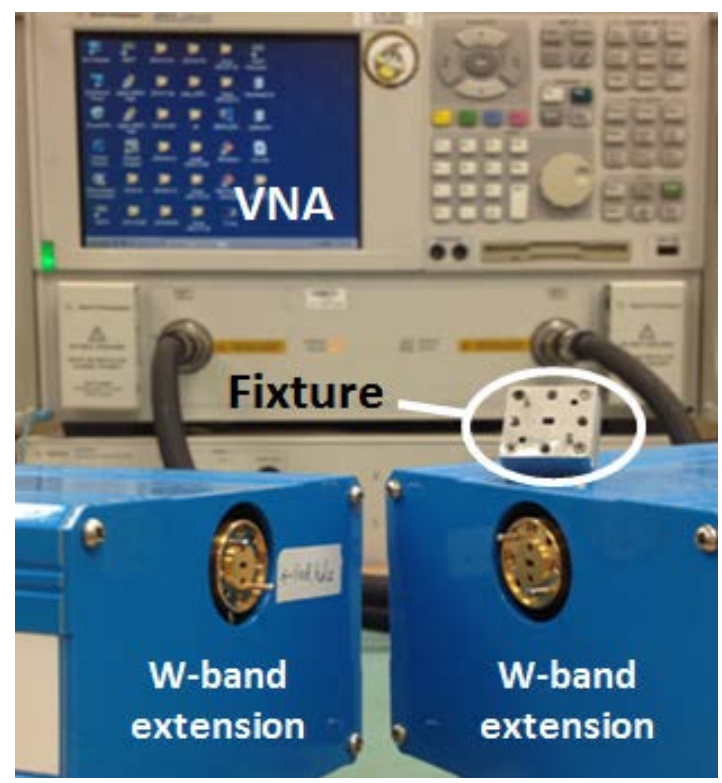

Figure 8. Test setup of the band-stop filter using VNA and W-band waveguide extensions.

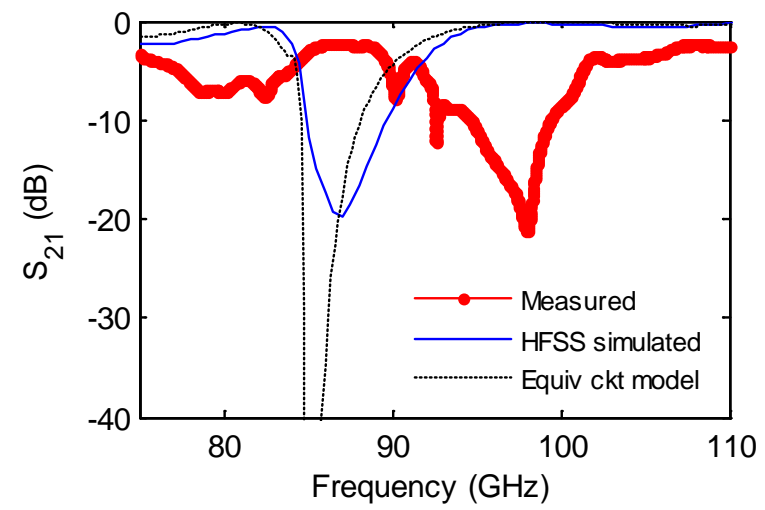

Figure 9. Measured vs. simulated $S_{21}(d B)$ of the EMXT band-stop filter.

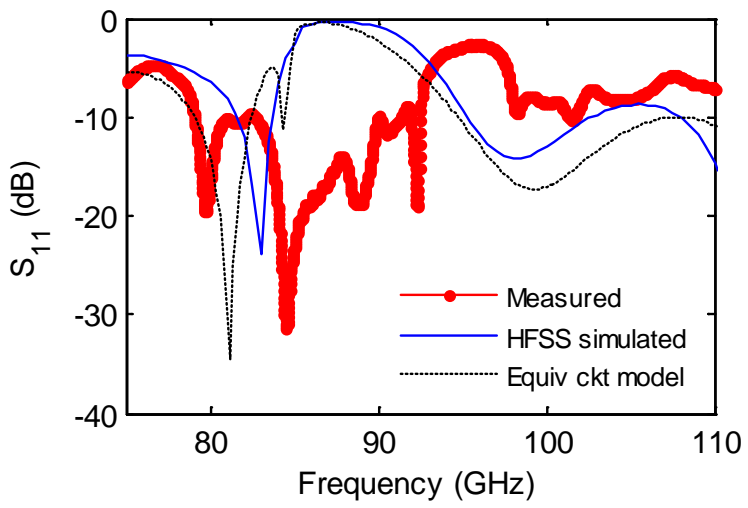

Figure 10. Measured vs. simulated $S_{11}(\mathrm{~dB})$ of the EMXT band-stop filter. 
fully demonstrated with a rejection band centered at $97 \mathrm{GHz}$ and a rejection level of 16 dB.

\section{References}

[1] Sievenpiper, D., Zhang, L., Broas, R., Alexopolous, N. and Yablonovtich, E (1999) HighImpedance Electromagnetic Surfaces with a Forbidden Frequency Band. IEEE Transactions on Microwave Theory, 47, 2059-2074. http://dx.doi.org/10.1109/22.798001

[2] Xin, H., Kim, M., Hacker, J.B., Higgins, J.A. and Rosker, M.J. (2002) Mutual Coupling Reduction of Low-Profile Monopole Antennas on High Impedance Ground Plane. Electronics Letters, 38, 849-850. http://dx.doi.org/10.1049/el:20020575

[3] Higgins, J., Kim, M., Hacker, J. and Sievenpiper, D. (1999) The Application of Photonic crystals to quasi-Optic Amplifiers. IEEE Transactions on Microwave Theory and Techniques, 47, 2139-2143. http://dx.doi.org/10.1109/22.798010

[4] Xin, H., Chen, T. and Kazemi, H. (2008) A W-Band Low-Loss Dual-Polarization QuasiTEM Waveguide. IEEE Transactions on Microwave Theory and Techniques, 56, 16611668. http://dx.doi.org/10.1109/TMTT.2008.924346

[5] Kariazidou, C., Contopanagos, H. and Alexopoulos, N. (2001) Monolithic Waveguide Filters Using Printed Photonic-Bandgap Materials. IEEE Transactions on Microwave Theory and Techniques, 49, 297-307. http://dx.doi.org/10.1109/22.903089

[6] Chew, S. and Itoh, T. (2001) PBG-Excited Split-Mode Resonator Bandpass Filter. IEEE Microwave and Guided Wave Letters, 11, 364-366. http://dx.doi.org/10.1109/7260.950762

[7] Xin, H., Higgins, A., Hacker, J., Kim, M. and Rosker, M. (2003) Electromagnetic Crystal (EMXT) Waveguide Band-Stop Filter. IEEE Microwave and Wireless Components Letters, 13, 108-110. http://dx.doi.org/10.1109/LMWC.2003.810121

[8] Eshrah, I., Kishk, A., Yakovlev, A., Glisson, A. and Smith, C. (2004) Analysis of Waveguide Slot-Based Structures Using Wide-Band Equivalent-Circuit Model. IEEE Transactions on Microwave Theory and Techniques, 52, 2691-2696. http://dx.doi.org/10.1109/TMTT.2004.837320

[9] Pan, P., Meng, F. and Wu, Q. (2008) A Composed Right/Left-Handed Waveguide with Open-Ended Corrugations for Backward-To-Forward Leaky-Wave Antenna Application. Microwave and Optical Technology Letters, 50, 579-582. http://dx.doi.org/10.1002/mop.23162

[10] Caloz, C. and Itoh, T. (2004) Electromagnetic Metamaterials: Transmission Line Theory and Microwave Applications. Wiley, New York. 
Submit or recommend next manuscript to SCIRP and we will provide best service for you:

Accepting pre-submission inquiries through Email, Facebook, LinkedIn, Twitter, etc. A wide selection of journals (inclusive of 9 subjects, more than 200 journals)

Providing 24-hour high-quality service

User-friendly online submission system

Fair and swift peer-review system

Efficient typesetting and proofreading procedure

Display of the result of downloads and visits, as well as the number of cited articles

Maximum dissemination of your research work

Submit your manuscript at: http://papersubmission.scirp.org/

Or contact cs@scirp.org 